\title{
Fetal antipsychotic exposure in a changing landscape: seeing the future
}

\author{
Kathryn M. Abe
}

\begin{abstract}
Summary
Pregnant women and their fetuses are increasingly likely to be exposed to antipsychotics. However, safety data remain limited. This editorial suggests that, in future, well-designed observational pharmaco-epidemiology is our best chance of illuminating risk for exposed
\end{abstract}

populations and of informing decision-making for women and clinicians.

\section{Declaration of interest}

None.
Kathryn M. Abel is Professor of Psychological Medicine \& Director Women's Mental Health, Institute of Brain, Behaviour and Mental Health at the University of Manchester. Her particular expertise and interests include maternal condition, parenting and offspring outcomes.

Pregnant women and their fetuses are more likely than ever to be exposed to antipsychotic medications particularly newer agents, which are increasingly used in women of reproductive age for a range of psychiatric disorders other than schizophrenia. ${ }^{1}$ Reproductive safety data remain surprisingly incomplete and guideline recommendations lend limited support to clinical risk-benefit analyses. $^{2-4}$ Randomised controlled trials of antipsychotic medication are considered unethical, and most available observational studies are underpowered, with biased samples unfit for purpose in a rapidly changing prescribing landscape. ${ }^{3}$ In a UK population approaching 66 million, 30004000 births per year are likely to be exposed to antipsychotics/ psychotropic medications. This editorial provides a critical summary of current knowledge about the risks of fetal antipsychotic exposure and proposes specifically how future observational epidemiology might fill crucial gaps in the evidence.

\section{Are we any clearer about exposure risks from first- and second-generation antipsychotics?}

\section{Congenital malformations}

Pregnancies of women with psychosis were originally shown to be twice as likely to result in congenital malformation or death, irrespective of chlorpromazine use in pregnancy when compared with well population controls. ${ }^{4}$ Offspring of women with psychiatric illness are highly likely to be at increased risk of neural tube defects because of higher rates of maternal obesity and reduced serum folate levels related to low dietary vitamin intake. Observational studies support this, ${ }^{2}$ but it remains uncertain whether antipsychotics cause any increased risk. ${ }^{5}$

Distinguishing the effects of maternal illness from the effects of medication is challenging. In the past, first-generation antipsychotics (FGAs) were mostly prescribed for morning sickness, not mental illness, using lower, intermittent doses. Meta-analysis of older studies report that infants of well mothers showed excess congenital malformation following exposure to phenothiazines $(n=2591)$ compared with unexposed infants $(n=71746)$ (odds ratio $(\mathrm{OR})=1.21,95 \%$ CI 1.01-1.45). ${ }^{6}$ However, estimates from recent cohorts may be more reliable because prevalence of outcomes in the reference population change over time. More recent Swedish data report possible excess (mainly cardiovascular) risk of congenital malformation $(\mathrm{OR}=1.52,95 \%$ CI 1.05-2.19) compared with well controls. ${ }^{7}$

In the UK, second-generation antipsychotics (SGAs) are now the most commonly prescribed antipsychotics. Manufacturers report no particular concerns for exposure in early pregnancy ${ }^{1,8}$ but manufacturer and spontaneous reporting data are biased by adverse outcomes reporting and often lack pregnancy outcomes. If we only take into account cases from unbiased but smaller samples and if we consider how long many of the compounds have been available, none of the antipsychotics appear to show themselves as clear teratogens. However, limb anomalies cannot be excluded with early exposure to haloperidol/penfluridol and there are no/few reports for aripiprazole, sertindole, amisulpride and zotepine. ${ }^{4}$

\section{Pregnancy outcomes}

More common outcomes allow clearer risk estimates in smaller samples, but even in population data, only small effects have been reported. In the largest sample exposed to any antipsychotic $(n=576)$, prematurity $(\mathrm{OR}=1.73,95 \%$ CI $1.31-2.29)$, birth weight $<2.5 \mathrm{~kg}(\mathrm{OR}=1.67,95 \%$ CI $1.21-2.29)$ and small-forgestational-age $(\mathrm{OR}=1.46,95 \%$ CI $0.99-2.15)$ are all increased. $^{7}$ A newer (2005-2009) Swedish cohort found higher risk in pregnancy-exposed infants of small-for-gestational-age $(\mathrm{OR}=$ 2.11, 95\% CI 1.29-3.47) and gestational diabetes $(\mathrm{OR}=2.78,95 \%$ CI 1.64-4.70), which disappears after adjustment for maternal factors such as smoking. ${ }^{9}$ Olanzapine or clozapine (grouped $n=169$ ) exposure was also associated with gestational diabetes $(\mathrm{OR}=2.39,95 \% \mathrm{CI} 1.12-5.13)$ and small-for-gestational-age ( $\mathrm{OR}=2.42,95 \%$ CI 1.24-4.70), but not large-for-gestational-age. The only study separating risk of drug from risk of illness is Lin et al: ${ }^{10}$ compared with the offspring of mothers with schizophrenia who were unexposed to FGAs, the infants of mothers with schizophrenia $(n=242)$ exposed to FGAs in early pregnancy were more likely to be premature $(\mathrm{OR}=2.46,95 \%$ CI $1.50-4.11)$, but not low birth weight, small- or large-for-gestational-age. However, they did not have information about important potential confounders, i.e. smoking, maternal weight, alcohol and substance use.

\section{Developmental outcomes}

There is a remarkable absence of studies of neonatal or developmental outcomes following pregnancy exposure to antipsychotics. Several cases of neonatal extrapyramidal syndrome have been reported with FGA exposure, ${ }^{8}$ but there are no other 
consistent patterns of adverse effects in the literature. Data are also limited for neurobehavioural sequelae of antipsychotic exposure; most data concern high-risk children of parents with psychosis. Several older studies following FGA-exposed infants suggest that deficits in infancy and early childhood have poor predictive value and disappear later in childhood. Older reviews failed to find differences in behavioural functioning or IQ up to 5 years (for example Altshuler et $a l^{6}$ ).

\section{Outstanding problems with the evidence base}

\section{The cohort effect}

For a number of reasons, the applicability of evidence gathered over a decade ago is questionable. First, the largest Swedish studies from 1994 to 2004 using midwife-data reported low psychosis prevalence in pregnant women $(<1$ in 1000$),{ }^{7}$ probably because midwives lacked awareness about psychosis. Contemporary Swedish cohorts (2005-2012) report prevalences of $>1$ in 1000 . Second, SGAs are now the most commonly prescribed antipsychotics in the UK, but earlier studies have limited SGA-exposure data. Finally, key outcomes such as rates of stillbirth or prematurity are affected by changing practice, which alters risk in the background population. Similarly, women becoming pregnant while on psychotropics are increasingly likely to be obese ${ }^{9}$ and, unlike Scandinavia, in the UK they are more likely to smoke. ${ }^{4}$ This is important because obesity and smoking independently increase risk of poor maternal and fetal outcomes.

\section{Illness v. medication}

Maternal (and in some cases paternal) psychotic disorder is consistently associated with adverse pregnancy outcomes such as congenital malformation, stillbirth, neonatal death, low birth weight and prematurity. ${ }^{11}$ However, lifestyle factors associated with severe illnesses/psychoses (smoking, substance misuse, poor nutrition and antenatal attendance) are independent risk factors for many of these outcomes (congenital malformation, prematurity, small- and large-for-gestational-age). Studies of the effect of maternal psychotropic exposure on pregnancy outcome often sample exposed mothers with a range of mental or other chronic illness severity that may confound findings. Lin et $a l^{10}$ is the only study to have analysed unexposed ill women, as well as unexposed well women. Unfortunately, their control group was mothers with schizophrenia who were not exposed to antipsychotics so that although they were able to examine excess risk of medication over and above illness alone, confounding by indication means that the ill, unexposed women may have been a less unwell group with less inherent risk than the ill, exposed group.

\section{Adherence to medication}

Evidence of adverse drug effects obviously must take account of adherence to medication, which is often compromised in severe mental illness. If an ill mother discovers she is pregnant, she may be especially likely to discontinue medication. Data-sets large enough to provide reliable information on pregnancy psychotropic exposure rarely contain detailed information on adherence, but recording prescription refills is one way to measure it. Some national registers, such as those used in the recent studies by Bodén et al, ${ }^{9,12}$ contain information on all prescription fills, including drugs dispensed using, amount, formulation and date of prescribing and dispensing.

\section{Antipsychotic groupings}

Prior literature confines analyses to exposure to all/any antipsychotics or, if numbers allow, to groupings of FGA/typicals and SGA/atypicals. Drugs in these groups differ in many of their properties including efficacy, side-effects and pharmacology and do not form a homogeneous class. ${ }^{9}$ Heterogeneity in effect and side-effects suggests that such 'improper grouping' may create confusion in the exposure.

\section{Confounding}

Compared with well, unexposed women, evidence suggests that a higher proportion of women exposed to psychotropics during pregnancy are immigrants, older, not living with the child's father, have more children and are smokers, with a high body mass index. ${ }^{9,11}$ These factors may all be independently associated with both exposure and outcome, acting as potential confounders of any association between drug and outcome in ill mothers. Few population studies account for potential confounders, which are unavailable in most data-sets. In addition, simultaneous administration of other agents is rarely considered because sample sizes or statistical complexity do not allow it and/or information on over-the-counter prescriptions is unavailable.

\section{Missing information}

No studies have been able to look separately at clozapine or the antipsychotics that are now most prescribed in the UK (olanzapine, quetiapine, risperidone). ${ }^{13}$ This is important because recent examination of the placental passage of antipsychotics (umbilical cord:maternal plasma concentration) suggests olanzapine has highest passage (mean $72.1 \%$, s.d. $=42.0$ ) and, also, higher rates of low birth weight and/or perinatal complications compared with other antipsychotics. ${ }^{14}$ In data-sets powerful enough, no studies examine quality of life, drug adherence or time to, or risk of, relapse in mothers with schizophrenia or related disorders who discontinue antipsychotic medication in pregnancy.

No long-term cognitive, psychopathological or developmental effects have been examined epidemiologically. This is particularly important because so-called 'high-risk' literature shows evidence that offspring of parents with severe mental illness are more likely to show poorer cognitive, social and clinical outcomes. ${ }^{11}$ However, none of this literature accounts for fetal psychotropic exposure.

Animal models are needed to explore the effects of antipsychotics on fetal growth and placental function, whereas multicentre studies might examine human placental handling of antipsychotics. Meanwhile, observational epidemiology offers far greater potential.

\section{What should future observational epidemiology} aim to do?

(a) Create cohorts large enough to enable analyses stratified by trimester of exposure and to examine individual drugs, including mono- $v$. polytherapy.

(b) Consider combining data-sets on common exposure and outcome variables (diagnoses, maternal characteristics, common obstetric outcomes).

(c) Adjust estimates for important potential confounders.

(d) Examine risk using both well and ill unexposed women as comparators.

(e) Link information obtained by midwives and self-reported medication use from antenatal visits, with actual filling of prescriptions during pregnancy to examine adherence. 
(f) Examine groups of drugs by side-effect profile. For example, susceptibility to metabolic side-effects such as maternal weight gain, gestational hypertension and gestational diabetes, and low-potency FGA/typicals should be grouped with -pines; thus, for example the 'typical' chlorpromazine grouped with 'atypical' olanzapine and clozapine.

(g) Compare outcomes between siblings and generations to account for non-measured family-wide effects.

(h) Examine evidence of longer-term developmental effects.

Kathryn M. Abel, MA, MBBS, MRCP, MRCPsych, PhD, Centre for Women's Health 3rd Floor Jean McFarlane Building, University of Manchester, Manchester M13 9PL, UK. Email: kathryn.abel@manchester.ac.uk

First received 11 Jul 2012, final revision 24 Oct 2012, accepted 31 Oct 2012

\section{Acknowledgements}

With thanks to Dr Robert Bodén, Professors Cecilia Magnusson, Christina Dalman and Pete Brocklehurst for useful discussions on the use of Scandinavian and other population samples in pharmaco-epidemiology.

\section{References}

1 McKenna K, Koren G, Tetelbaum M, Wilton L, Shakir S, Diav-Citrin O, et al. Pregnancy outcome of women using atypical antipsychotic drugs: a prospective comparative study. J Clin Psychiatry 2005; 66: 444-9.

2 Webb RT, Howard L, Abel KM. Antipsychotic drugs for non-affective psychosis during pregnancy and postpartum. Cochrane Database Syst Rev 2004: 2: CD004411.
3 National Institute for Health and Clinical Excellence. Antenatal and Postnatal Mental Health. Clinical Guideline 45. NICE, 2007.

4 Barnes TR. Evidence-based guidelines for the pharmacological treatment of schizophrenia: recommendations from the British Association for Psychopharmacology. J Psychopharmacol 2011; 25: 567-620.

5 Webb RT, Pickles AR, King-Hele SA, Appleby L, Mortensen PB, Abel KM. Parental mental illness and fatal birth defects in a national birth cohort. Psychol Med 2008; 38: 1495-503.

6 Altshuler LL, Cohen L, Szuba MP, Burt VK, Gitlin M, Mintz J. Pharmacological management of psychiatric illness during pregnancy: dilemmas and guidelines. Am J Psychiatry 1996; 153: 592-606.

7 Reis $M$, Kallen B. Maternal use of antipsychotics in early pregnancy and delivery outcome. J Clin Psychopharmacol 2008; 28: 279-88.

8 Gentile S. Antipsychotic therapy during early and late pregnancy. A systematic review. Schizophr Bull 2010; 36: 518-44.

9 Bodén R, Lundgren M, Brandt L, Reutfors J, Kieler H. Antipsychotics during pregnancy: relation to fetal and maternal metabolic effects. Arch Gen Psychiatry 2012; 69: 715-21.

10 Lin $\mathrm{HC}$, Chen IJ, Chen YH, Lee HC, Wu FJ. Maternal schizophrenia and pregnancy outcome: does the use of antipsychotics make a difference? Schizophr Res 2010; 116: 55-60.

11 Abel KM, Morgan VA. Mental illness, women, mothers, and their children. In Textbook in Psychiatric Epidemiology (3rd edn) (eds MT Tsaug, M Tohen, P Jones): 483-514. Wiley-Blackwell, 2011.

12 Bodén R, Lundgren M, Brandt L, Reutfors J, Andersen M, Kieler H. Risks of adverse pregnancy and birth outcomes in women treated or not treated with mood stabilisers for bipolar disorder: population based cohort study. BMJ 2012; 345: e7085

13 The Health and Social Care Information Centre. Prescription Cost Analysis: England 2010. The Health and Social Care Information Centre 2011.

14 Newport DJ, Calamaras MR, DeVane CL, Donovan J, Beach AJ, Winn S, et al. Atypical antipsychotic administration during late pregnancy: placental passage and obstetrical outcomes. Am J Psychiatry 2007; 164: 1214-20.

\section{Poems by doctors}

\section{Ode to Anxiety}

\section{Ankur Sharma}

Whine of mangled nerves Like convoluting steel rails conducting trains of worry Deafeningly.

Blur of contracting sight

Like curtains falling

Leaden lids, shutting down Hazily.

Anxiety, you are potent

Like cyanide

choking on contact

Gaspingly.

Leave me, leave me

Like life from a corpse

Stop this galloping pulse

Hurriedly.

(c) Ankur Sharma 